\title{
A Highly-reliable Smart Kitchen Security Alarm System Based on Internet of Things
}

\author{
Yun Wang ${ }^{1}$, Tong Zhou ${ }^{1, \text { a, * }}$ \\ ${ }^{1}$ School of Mechanical Engineering , Nanjing University of Science and Technology, Nanjing \\ 210094, China. \\ azhoutong@njust.edu.cn
}

Keywords: Fuzzy reasoning, Temperature and humidity compensation, Internet of things, Intelligent

\begin{abstract}
Aiming at the shortcomings of high false alarm rate, single alarm mode and low degree of intelligence for gas leakage alarms currently on the market, a high reliability and intelligent alarm system for kitchen safety is proposed. By establishing a mathematical model of temperature and humidity compensation for gas sensors, this method compensates the nonlinearity error caused by temperature and humidity based on fuzzy reasoning, and improves the detection precision and reduces the false alarm rate. On this basis, the use of Internet of Things technology to achieve multi-system alarm and intelligent protection, improves the system's security and reliability and intelligence, and takes the dynamic threshold for different users of the kitchen environment to take the difference, greatly reducing the system's false positive rate.
\end{abstract}

\section{Introduction}

With the continuous improvement of people's living standards, the home environment has gradually been replaced by the smoke-free era. The use of liquefied gas, natural gas, and electricity in the kitchen is becoming more and more common, and the security risks are also increasing ${ }^{[1]}$.In particular, poisonous and harmful gases such as carbon monoxide and methane produced by the combustion of liquefied gas and natural gas are kitchen invisible killers. And if the open fire in the kitchen is also easy to be neglected, a more serious fire will be caused and the building and personal safety will be caused. In order to be able to know the air condition of the kitchen in a timely manner and ensure the safety of life and property, the safety monitoring of the kitchen has become imminent. Therefore, it is of great significance to adopt an intelligent kitchen safety monitoring system. Adopting the Internet of Things ${ }^{[2]}$ to monitor the safety of the kitchen and achieve multiple alarms will greatly improve the efficiency and reliability of the system.

Gas detection and alarm system plays an increasingly important role in people's daily life and various fields ${ }^{[3]}$. For the detection of toxic gases such as methane, MQ-2 smoke sensors are widely used due to their high detection sensitivity, fast response, and good stability due to the use of 
semiconductor gas sensors as sensing elements. However, according to actual experimental results and experience, it is shown that the semi-conductor type gas sensor floats in the air when the intrinsic resistance is relatively obvious, and the main environmental factors that cause drifting are the influence of temperature and humidity. Therefore, while the MQ-2 sensor is used as the main module of the concentration detection, a temperature and humidity compensation method based on fuzzy reasoning is proposed to improve the accuracy of concentration detection and reduce the false alarm rate.

In terms of system design, the system proposed in this paper separates the detection control end from the natural gas pipeline on/off control end, adopts infrared wireless control, reduces line faults, and achieves intelligent protection. Through the Internet of Things technology, the status of the kitchen is fed back to the remote server, and the server automatically implements an alarm route including calling the owner of the phone and informing the community management personnel. In order to improve reliability, while performing temperature and humidity compensation at the time of detection, dynamic thresholds are used when different environments of different kitchen environments are used, and different kitchens have different concentration thresholds, so as to intelligently reduce the false alarm rate.

\section{A Temperature and Humidity Compensation Method Based on Fuzzy Reasoning}

\subsection{Temperature and humidity compensation method based on fuzzy reasoning}

The MQ-2 sensor adopts a semiconductor gas sensor as a detection element, and it floats more obviously in the intrinsic resistance of the general detection environment, and the main factor in the generation of drift is temperature and humidity. In order to reduce the false alarm rate of the system, the temperature and humidity compensation must be designed. However, because the intrinsic resistance of the semiconductor gas sensor is nonlinear in the characteristic curve of temperature and humidity, the temperature and humidity data can not be used to compensate the temperature and humidity directly through the temperature and humidity data signal, so a fuzzy reasoning method is proposed to deduce and modify the temperature and humidity.

Fuzzy reasoning is the use of fuzzy mathematics ideas and theories to deduce the measured data as input, first fuzzy the measured data, and then through the "if ... and ... then ..." inference rules to get the output, and specific The output is mainly based on actual experience and experimental data.

The temperature and humidity compensation based on fuzzy inference is mainly shown in Figure 1. Firstly, through the MQ-2 sensor and the temperature and humidity sensor, the initial concentration signal and temperature and humidity signal are obtained respectively, and the corresponding value of the natural gas concentration is obtained through a mathematical operation. The temperature and humidity signals are input to the system as input terminals of the fuzzy inference system. The fuzzy inference system fuzzifies the input quantities $\mathrm{T}$ and $\mathrm{H}$ into six different degree variables, namely VB (large), B (large), MB (upper middle), MS (lower middle), and S (small)), VS (very small) six variables, through the "if ... and ... then ..." inference rules to get the output level variables. Then through the output level variable through the experimental data to obtain the voltage correction coefficient $\alpha$, the output voltage can be calculated by the following formula:

$$
V_{C^{\prime}}=V_{C} \times \alpha=V_{C C} \times \frac{R}{R+R_{M 2}} \times \alpha=\frac{5 \alpha R}{R+1}
$$


In the formula, $V_{C^{\prime}}$ is the correction voltage, $V_{C C}$ is the supply voltage $5 \mathrm{~V}, \mathrm{R}$ is the equivalent element of the detection element, $R_{M 2}$ is the load resistance, and the resistance value is $1 \mathrm{k} \Omega$.

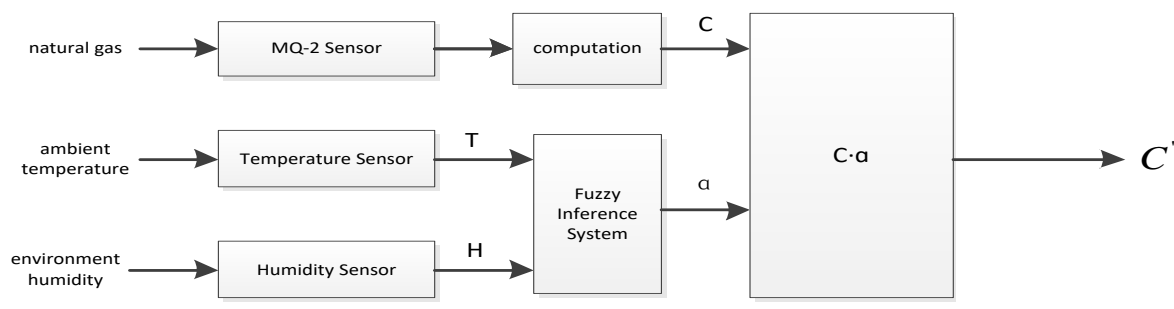

Fig 1. Temperature and humidity compensation based on fuzzy reasoning

\subsection{Temperature and humidity compensation coefficient experiment}

By the temperature in accordance with $-5^{\circ} \mathrm{C}$ to $5{ }^{\circ} \mathrm{C}$ for VS, $5{ }^{\circ} \mathrm{C}$ to $15^{\circ} \mathrm{C}$ for $\mathrm{S}, \ldots, 45^{\circ} \mathrm{C}$ to $55^{\circ} \mathrm{C}$ for the VB calibration; the humidity in accordance with $25 \mathrm{RH}$ to $35 \mathrm{RH}$ for VS, 35RH to $45 \mathrm{RH}$ for $\mathrm{S}, \ldots, 75 \mathrm{RH}$ to $85 \mathrm{RH}$ are VB calibrations, which blur the temperature and humidity signals to obtain a fuzzy inference table.

For the alarm system, the threshold actually detected is relatively small relative to the maximum value that can be detected by the sensor. In other words, R1 is much smaller than $\mathrm{R} 2$, and it can be concluded through experiment that the voltage compensation coefficient is less than 1.5, according to formula (1) can be calculated as follows:

$$
V_{m^{\prime}}=\alpha \times \frac{R_{M 2}}{R_{M 2}+R_{S}} \times V_{C C} \approx \frac{R_{M 2}}{R_{M 2}+\frac{R_{S}}{\alpha}} \times V_{C C}
$$

It can be concluded that the voltage compensation coefficient can almost be regarded as the inverse ratio of the change coefficient of the resistance when the alarm is performed. Therefore, it can be seen that when using the voltage compensation coefficient to perform the compensation experiment, the concentration feedback can be performed more accurately.

According to the principle of MQ-2, the experiment can not be carried out under certain concentration of natural gas, so the resistance of the detection element is often up to $20 \mathrm{k} \Omega$, and the actual measured voltage is less than $0.1 \mathrm{~V}$, resulting in a large error in the measurement result. Therefore, the load resistance of the sensor is amplified to $20 \mathrm{k} \Omega$, and the experiment is performed by measuring the voltage across the load resistor.

Table 1. fuzzy inference table

\begin{tabular}{ccccccc}
\hline TH & VB & B & MB & MS & S & VS \\
\hline VB & 1.46 & 1.29 & 1.15 & 1.03 & 0.96 & 0.90 \\
B & 1.37 & 1.20 & 1.09 & 0.98 & 0.92 & 0.87 \\
MB & 1.28 & 1.14 & 1.03 & 0.95 & 0.89 & 0.85 \\
MS & 1.23 & 1.10 & 1.00 & 0.92 & 0.87 & 0.84 \\
S & 1.20 & 1.06 & 0.97 & 0.90 & 0.85 & 0.82 \\
VS & 1.16 & 1.03 & 0.94 & 0.89 & 0.84 & 0.81 \\
\hline
\end{tabular}

The experiment was conducted in a temperature and humidity test chamber, and the temperature and humidity were adjusted. Different fuzzy variables were used to take the preset intermediate values, that is, the temperature was $0^{\circ} \mathrm{C}, 10^{\circ} \mathrm{C}, 20^{\circ} \mathrm{C}, 30^{\circ} \mathrm{C}, 40^{\circ} \mathrm{C}, 50^{\circ} \mathrm{C}$, and the humidity was $30 \mathrm{RH}$, $40 \mathrm{RH}, 50 \mathrm{RH}, 60 \mathrm{RH}, 70 \mathrm{RH}, 80 \mathrm{RH}$, this experiment takes $20^{\circ} \mathrm{C}, 60 \mathrm{RH}$ state is the standard parameter, 
set the voltage compensation coefficient is 1.00 , carry on the experiment and calculate the voltage compensation coefficient table as shown in Table 1.

\section{Alarm System Hardware Design}

The hardware design of the system designed in this paper includes constant potential circuit, filter circuit, AD conversion circuit, MCU main control circuit and power supply module. Finally, the GPRS module ${ }^{[4]}$ sends the detection data to the remote detection terminal. The system MCU selects ST's STM32F103C8T6 as the main control chip, and the schematic diagram of its structure is shown in Figure 2. When the system is working, the detection end is located at the ceiling above the burning appliance. After the initial detection signal is filtered by the sensor, it passes through the AD conversion circuit and sends the digital data to the MCU. the temperature and humidity signals are fuzzy, sent to the fuzzy inference system, and then calculated, and the parameters such as the detected concentration and the state of the electric valve are periodically sent to the remote TCP server through the GPRS module. At the same time, the MCU has an acousto-optic alarm and infrared communication module. When the detected concentration is greater than the threshold, the MCU controls the sound and light alarm to alarm, and wirelessly controls the electric valve at the natural gas pipeline through the infrared sending module ${ }^{[5]}$ to achieve the cutoff of natural gas. The actual designed PCB circuit board is shown in Figure 3.

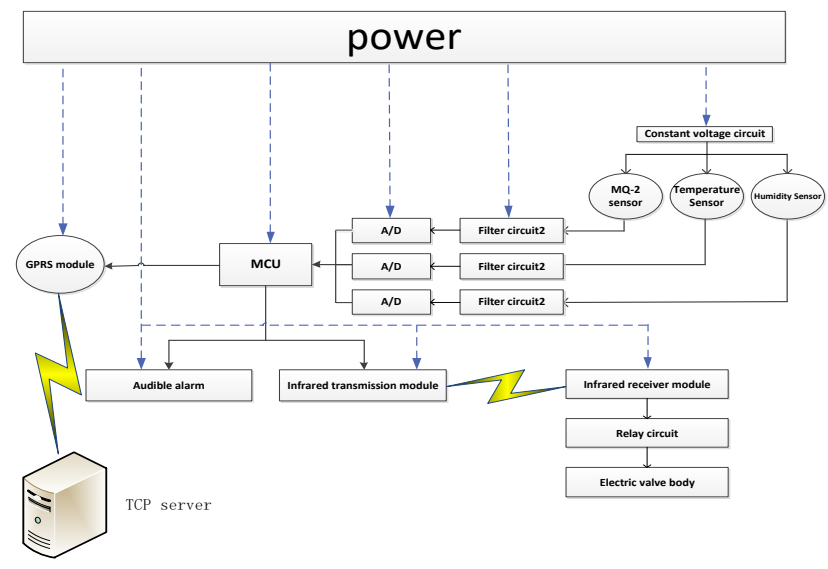

Fig 2. Hardware structure diagram of smart kitchen security alarm system

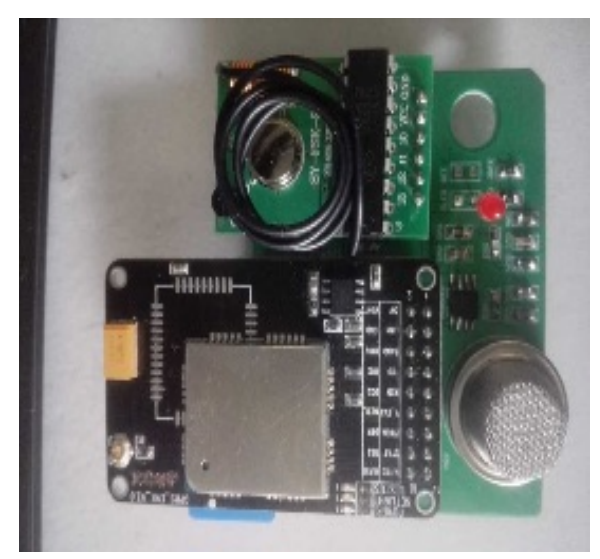

Figure 3. system test control end PCB physical map 


\section{Alarm System Software Design}

The software design of the alarm system is mainly based on the STM32F103 microcontroller as the core of the program design, which mainly includes $\mathrm{AD}$ conversion program, serial communication program, temperature and humidity compensation algorithm, alarm program, interrupt program, threshold update program, to achieve the system's own operation. The overall flow of the program is shown in Figure 4. After the system is powered on, the MQ-2 sensor works immediately and reads the data collected by the MQ-2 sensor and temperature and humidity sensor every 5 seconds. Then, through the fuzzy inference system, the voltage compensation coefficient is obtained and calculated, and the corrected concentration is compared with the dynamic threshold. When the alarm is exceeded, the alarm loop is entered, the valve body is closed, the sound and light alarm is sent, and the concentration exceeds the directive is sent to the TCP server. The server notifies the user by telephone, contact the person in charge and other alarm methods. After the alarm is released, the server can be reset remotely. In general, the average concentration value is sent to the server every 3 minutes. Configure interrupt routines, the server can send higher-level instructions to control the system state. The interface between the system detection end and the server adopts a special interface protocol for data generation. A threshold update program is also provided to automatically modify natural gas concentration thresholds in different kitchen environments to improve system reliability and reduce false alarm rates.

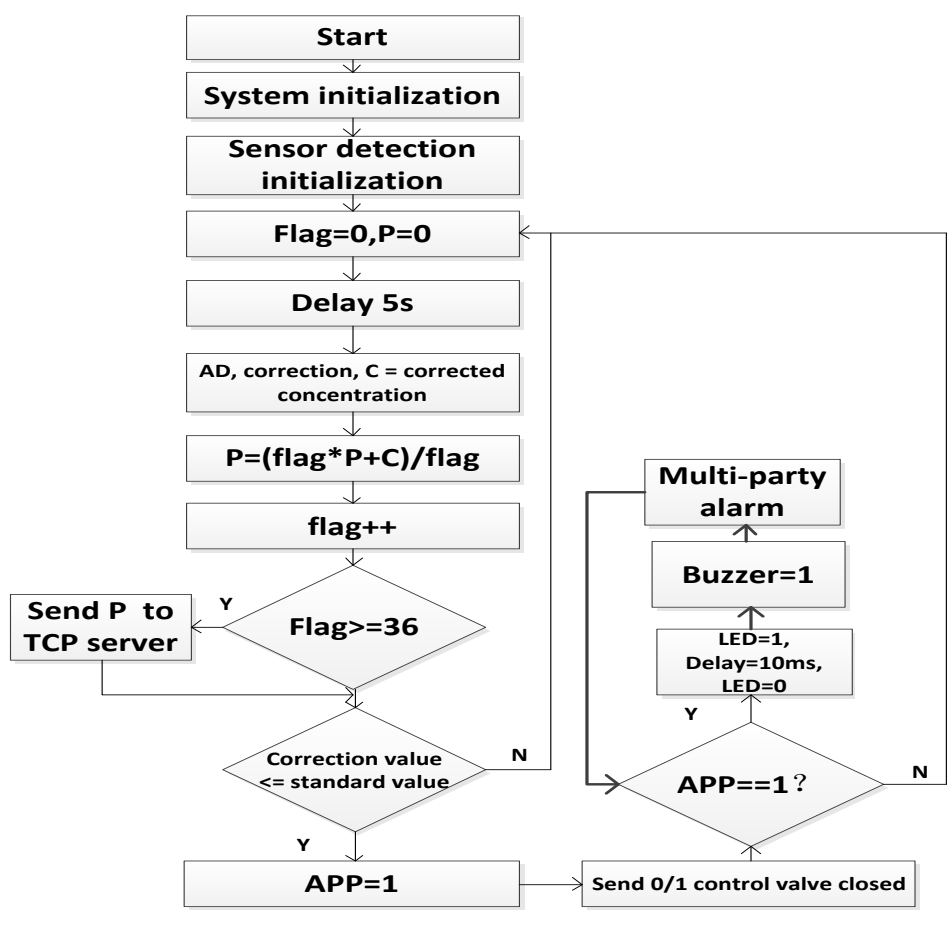

Figure 4. program logic diagram

\section{Summary}

The design and implementation of a highly reliable smart kitchen security alarm system based on the Internet of Things has been successfully demonstrated and discussed. The system overcomes the shortcomings of low intelligence and high rate of false alarms for gas leak alarms currently on the market. With the Internet of Things as a medium, the situation of kitchens within a certain area is 
summarized and managed in a unified manner, which will greatly reduce occurrence of kitchen accidents. The system not only uses the temperature and humidity compensation algorithm to improve the detection accuracy of the system, but also adopts the separation of the electric valve and the detection control end, which reduces the complexity of the circuit distribution and improves the reliability of the system. The actual experiment shows that the system has fast feedback and high reliability.

\section{References}

[1] Zhijie Tang, et al. Remote Alarm Monitor System Based On GSM and ARM, J. Elsevierjournal, 2011, 814:65-69.

[2] Mussab Alla,, et al.A Review of Smart Home Applications based on Internet of Things, J. Elsevierjournal, 2017, 11,01:48-65

[3] Kuang Gang Fan , Xin Zhang, et al.Design and Implementation of the Security Subsystem in the Smart Home System, J.Trans Tech, 2013, 427:1254-1257.

[4] Ning Ning Song, Ze Tao Li, et al. Sensor Network and the Internet Data Transmission Technology Research Based on GPRS, J.Trans Tech,2013,346:59-64.

[5] Xiu Jiao Jiang, Remote Access and Control System Based on Infrared Communication Technology, J. Trans Tech, 2013,380: 3575-3579. 